\title{
Recovering an Operating Room Schedule during a Global Pandemic
}

\section{A Method for Safe and Swift Increases in OR Volume during Times of Crisis}

\author{
Ryan Brumit ${ }^{1}$ (i) $\cdot$ Bethany Daily ${ }^{2} \cdot$ Wilton C. Levine ${ }^{3}$ (i) \\ Received: 14 October 2020 / Accepted: 1 December 2020 / Published online: 7 January 2021 \\ (C) The Author(s), under exclusive licence to Springer Science+Business Media, LLC part of Springer Nature 2021
}

As the SARS-CoV2 pandemic surged in March 2020, hospitals across the world worked tirelessly to defer all non-essential surgical procedures in an effort increase hospital capacity, conserve personal protective equipment (PPE), and free up Nursing and Anesthesia staff and peri-operative space for redeployment and care of SARS-CoV2 patients. This reduction was both out of necessity to care for incoming SARS-CoV2 patients and also in collaboration with state governments that mandated significant reductions in elective procedures [1]. This dramatic reduction of the operating room schedule was unprecedented, and within a matter of four weeks, Massachusetts General Hospital (MGH) had deferred approximately 6500 surgeries and was operating at $15 \%$ of its average pre-COVID surgical volume. This nadir lasted until May 11, 2020, at which point the Massachusetts Department of Public Health (DPH) lifted the restrictions on the types of procedures that could be performed, and the hospital was given the green light to ramp up its OR schedule [2]. In the months that followed, MGH developed a method for safe and swift recovery of the OR schedule, which helped meet the clinical needs of many thousands of patients awaiting surgery, and the business needs of a hospital that suspended most all revenue-generating procedures. The methodology we describe dovetails the methodology found in Surgical Case Deferment During a Global Pandemic [3], and has applicability for our current pandemic, as well as during

This article is part of the Topical Collection on Patient Facing Systems

Ryan Brumit

rbrumit@partners.org

1 Perioperative Program Development, Perioperative Services, Massachusetts General Hospital Boston, Boston, MA, USA

2 Perioperative Service and Healthcare Systems Engineering, Massachusetts General Hospital, Boston, MA, USA

3 Perioperative Services and Medical Procedure Areas, Department of Anesthesia, Critical Care and Pain Management, Massachusetts General Hospital, Boston, MA, USA other emergency situations such as blizzards, hurricanes or massive power outages.

\section{Stakeholders and scope}

In the early stages of the pandemic, Surgical and Perioperative leadership developed the following Triage Categories in an effort to work within the Massachusetts DPH guidelines of acceptable procedures and provide safe and reasonable care for patients:

1. Life and limb saving procedures

2. Treatment of life threatening / unstable disease

3. Treatment of infections that would otherwise become life threatening

4. Cancer

a. Cancers in patients who have completed their neoadjuvant therapy and are in the window of resectability, and for whom non-operative temporizing maneuvers are not possible

b. Aggressive cancers that will grow significantly in 2 months for which other therapies cannot be used to temporize (e.g. triple-negative breast cancer)

c. Second part of staged procedures in which the first stage has been completed (e.g. patient has an open wound awaiting reconstruction)

d. Diagnostic procedure required to allow initiation of appropriate cancer therapy (e.g.diagnosis of lymphoma or diagnosis of metastatic cancer)

5. Acute symptoms (e.g. GI bleeding, bowel obstruction, dysphagia and/or aspiration risk, airway encroachment) for which alternative therapy is not appropriate

6. Fractures needing surgical repair.

7. Cancer that had been previously deferred

8. New cancer needing surgical or interventional procedure

9. Functionally limiting disease, or, Chronic progressive disease 
10. Stable chronic disease

11. Screening disease

12. Cosmetic

During the moratorium on elective procedures, our institution only provided surgical and procedural care for patients in categories 1-6 above. As the moratorium relaxed and state guidelines changed, we worked to systematically add procedures for patients in class 7-12.

Implementation of these categories and the associated recovery mythology requires a multidisciplinary team. MGH has leveraged the following stakeholders to aid in the OR schedule recovery process:

- Perioperative Services Administration: development and facilitation of method steps

- Medical Director: ownership of the method and management of the OR schedule

- Surgical Chiefs: ownership of service recovery and triage

- Surgeons/Surgical Offices: practice-level prioritization and patient communication

- Central Scheduling: placement of all surgical cases

- IS/EHR Resources: development and distribution of standard reporting tools

In addition to the role groups directly responsible for developing and carrying out the recovery methodology, the rate at which the schedule can be recovered is contingent the gradual return of Nursing and Anesthesia staff from the units to which they were previously deployed. The simultaneous ramp down of the COVID ICUs and the ramp up of the surgical schedule must be carefully orchestrated, as to not leave either function under-supported.

\section{Triaging thousands}

In order to determine a patient triage order, it was necessary to categorize each deferred procedure as one of the above categories. Since we had continued to provide care for categories 1-6, our task was to assign each of our 6500 deferred cases into our lower six categories (numbers $7-12$ in the classification list). Once complete, we would effectively have a patient and case triage priority order. The Mass General Brigham (MGB) procedure dictionary contains approximately 7500 unique procedures, far too many to efficiently map to the six Triage Categories. Through discussion with the enterprise team that supports the MGB electronic health record (EHR), an alternative solution was identified. The Healthcare Cost and Utilization Project (HCUP), a Federal-State-Industry partnership sponsored by the Agency for Healthcare Research and Quality, developed the Clinical
Classification Software (CCS), "a tool for clustering patient diagnoses and procedures into a manageable number of clinically meaningful categories [4]." This tool had already been incorporated into MGB's EHR, which bundled each of the 7500 unique procedures into one of 160 CCS Procedure Groupings.

These Procedure Groupings were then assigned a Triage Category by Perioperative Services Administration, with the understanding that they were thoughtful recommendations to help streamline the thorough clinical review that was ultimately required. From there, a complete list of deferred cases was pulled from the EHR. This dataset included cases that were previously scheduled and deferred, as well as new deferrable cases that had been entered into the EHR since the elective case deferment directive took effect in March 2020. The file contained all of the relevant case booking datapoints to help make decisions around priority, including: Primary Procedure, CCS Procedure Grouping, Pre-Op Diagnosis, Patient Classification, etc.

Using basic data management formulas, each of the 6500 deferred surgical cases was assigned a Triage Category recommendation by leveraging the CCS Procedure Group that was linked to the Primary Procedure. The master case file was then separated into service-specific lists, which were then distributed to the relevant Surgical Chiefs, who were asked to conduct a clinical review of each surgical case and its corresponding Triage Category. If their assessment of the Triage Category was different than the initial recommendation, they were instructed to update the file accordingly and return to Perioperative Services Administration upon completion of their review (Fig. 1a).

Mapping Sample:

\begin{tabular}{lll}
\hline $\begin{array}{l}\text { EHR Procedure } \\
\text { Name }\end{array}$ & $\begin{array}{l}\text { CCS Procedure } \\
\text { Group }\end{array}$ & $\begin{array}{c}\text { Recommended } \\
\text { Triage Category }\end{array}$ \\
\hline $\begin{array}{l}\text { ARTHROPLASTY } \\
\text { TOTAL HIP }\end{array}$ & $\begin{array}{c}\text { 153-HIP } \\
\text { REPLACEMENT; } \\
\text { TOTAL AND }\end{array}$ & STABLE CHRONIC \\
COLECTOMY & PARTIAL & \\
& 078-COLORECTAL & CANCER - \\
& RESECTION & PREVIOUSLY \\
PLACEMENT DRAIN & 004-DIAGNOSTIC & DEFERED \\
LUMBAR SPINE & SPINAL TAP & PROGRESSIVE OR \\
& & FUNCTIONALLY \\
REVISION & 028-PLASTIC & LIMITING DISEASE \\
RHINOPLASTY & PROCEDURES ON & \\
& NOSE & \\
SEGMENTECTOMY & 036-LOBECTOMY OR & CANCER - \\
LUNG WITH & PNEUMONECTOMY & PREVIOUSLY \\
BRONCHOSCOPY & OR OTHER & DEFERRED \\
FLEXIBLE & EXCISION OF LUNG & \\
& TISSUE & \\
TRANSPLANTED & 105-KIDNEY & CHRONIC \\
KIDNEY & TRANSPLANT & PROGRESSIVE OR \\
REMOVAL & & FUNCTIONALLY \\
& & LIMITING DISEASE \\
\hline & & \\
& &
\end{tabular}


Fig. 1 Overview of the $\mathrm{MGH}$ surgical case recovery method and its impact on operating room volume. a) Flowchart for surgical case recovery method. b) AMC Surgical Case Volume - Pre and Post-Recovery Monthly Trend c) AMC \& ASC Surgical Case Volume - Recovery Window Weekly Trend Weekly Trend a

Deferred Case Recovery Workflow
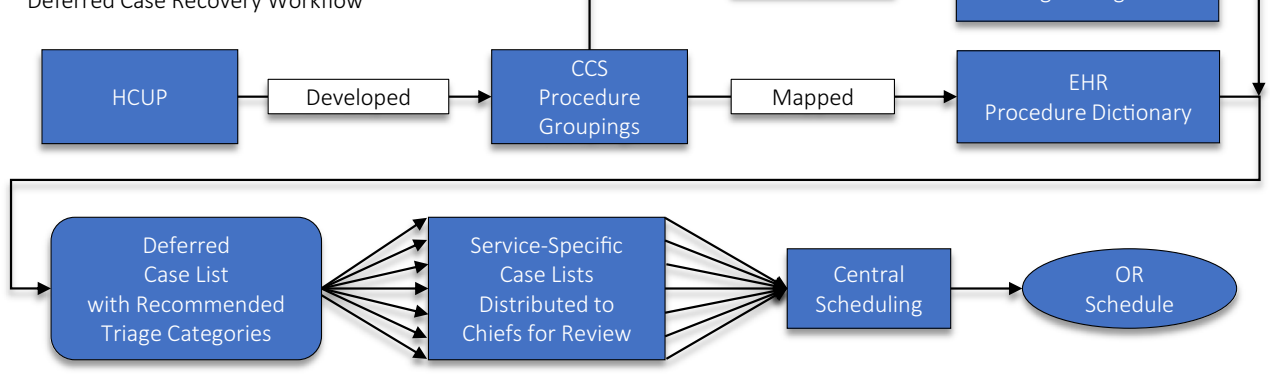

b

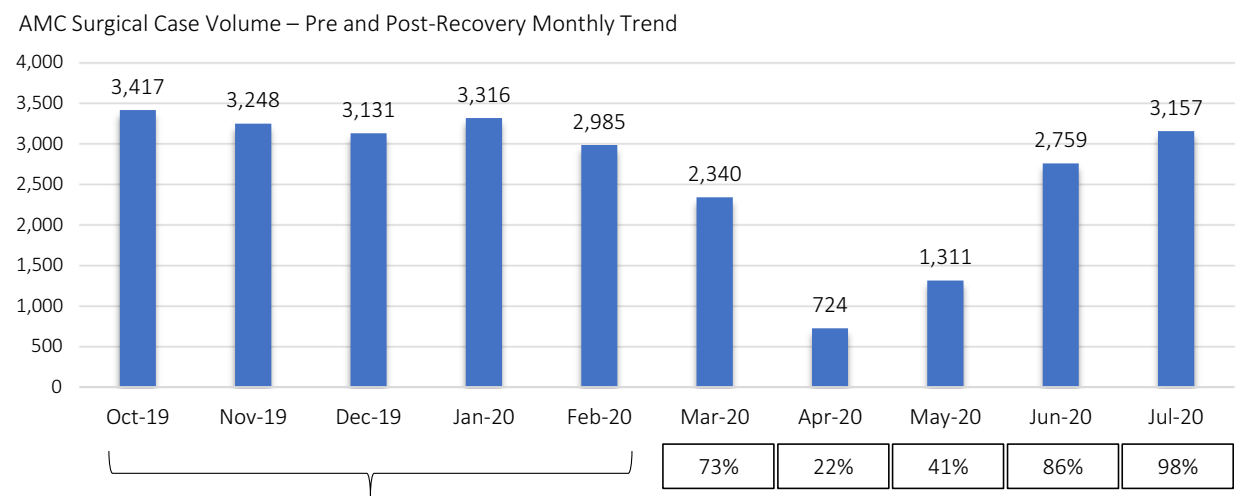

Pre-Pandemic Average Month Case Volume = 3,219

$\%$ of Pre-Pandemic Average Month Case Volume

C

AMC \& ASC Surgical Case Volume - Recovery Window Weekly Trend

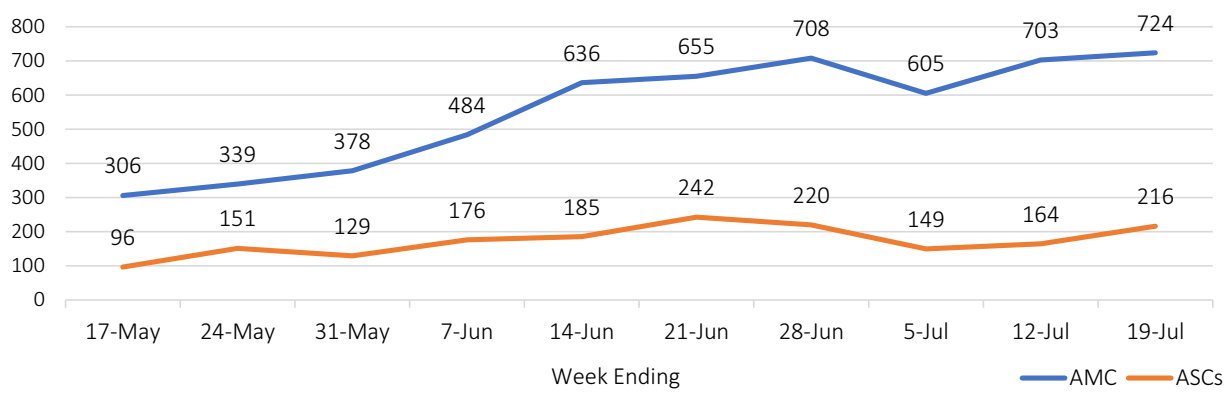

\section{Recovering the scheduling}

In the eight weeks between May 11th and July 5th, MGH ramped up from 10 to 58 rooms running each day. This required a significant amount of flexibility from all stakeholders, as the strategies for common operating room operational management factors such as block time allocation, case placement, patient coordination and pre-operative COVID testing were in a constant state of flux due to the everevolving nature of the SARS-CoV2 pandemic. Additionally, each component of the recovery strategy overlapped significantly, which would often send ripples through the various workflows any time adjustments were made. In spite of these operational and logistical challenges, a quick recovery was necessary to ensure that patients who had been deferred but could no long be delayed had access to receive care. As concluded by Sud et al. (2020) that even "modest delays in surgery for cancer incur significant impact on survival [5]."

Prior to the SARS-CoV2 pandemic, the vast majority of OR blocks were assigned to specific surgeons. Additionally, the pre-pandemic MGH surgical scheduling structure was a mix of centralized and decentralized, where some surgical services placed cases directly onto the OR schedule, whereas other services went through the hospital's centralized scheduling team to have their cases placed. Immediately after the hospital was charged with deferring all non-essential procedures back in mid-March, decisions were made to assign all blocks at the service level and to centralize all case placement through central scheduling. Over the course of the recovery phase, these changes proved to be incredible beneficial for 
ramp-up planning, as well as the organization of the daily OR schedule.

Service-level block allocation was initially based on anticipated essential case volume. However, around the time when the OR schedule reached approximately 50\% capacity, the strategy shifted to allocate blocks to services based on their percentage of allocated time on the pre-pandemic block schedule. This was a way to smoothly transition back into a fair and balanced distribution of a full-capacity OR schedule.

In terms of actually filling the OR schedule during the recovery phase, each surgical service was asked to internally prioritize specific patients based on clinical need and upcoming assigned block time. For most services, this meant the Surgical Chief worked with their surgeons to review the previously generated deferred case list and corresponding Triage Category recommendations, ultimately determining where and when each patient should be scheduled. As the recovery phase progressed and the number of service-assigned blocks increased, this approach became quite difficult for each service leadership team to sustain; the volume of cases that needed to be prioritized was simply too high to be thoughtfully reviewed and approved centrally within each service. As such, a decision was made to transition back to surgeon-assigned blocks when $100 \%$ capacity was reached. Surgeon-assigned blocks allowed each blockholder to 'decentrally' prioritize their own patients as they see fit.

Every component of this methodology requires a significant amount of communication across the various stakeholders and team members. Noteworthy channels include: a single pathway for disseminating new information as soon as it becomes available, daily collection of feedback from the front lines that's routed back to the command center, and transparency between all enterprise OR locations and procedural areas to spread best practices. Recurring virtual meetings were held to facilitate with these communication channels - they always started on time, were run with expert efficiency and only occurred with a frequency that aligned with the needs of its attendees.

\section{Leveraging ambulatory surgery centers}

Soon after the March 15th order from the DPH to defer all non-essential surgeries, the MGH Ambulatory Surgery Centers (ASC) temporarily suspended operations and redeployed staff and equipment to the Main Campus Academic Medical Center (AMC) to support the escalating needs. However, about two months later in the early stages of the recovery phase, when the AMC's inpatient capacity remained mostly dedicated to COVID-positive and COVID-risk patients, the ACSs became an invaluable resource for new non-deferrable and previously deferred Day Surgery cancer patients (e.g. breast, thyroid, melanoma). After just a week of planning, and gathering previously redeployed staff and borrowed equipment, the eight ORs at the MGH Danvers
ASC were brought back into operation. Not long after the Danvers ASC was brought back online, the Waltham ASC reopened its four orthopedic ORs within a similar timeline.

\section{Summary}

As of October 1st, 2020, the MGH AMC OR continues to run 58 rooms daily and completes an average 750 surgical cases per week, which satisfies the pre-pandemic definition of a full schedule. This is a result of an enormous multidisciplinary effort focused on safe and swift assembly and alignment of patients, clinicians, OR space and equipment. Such a significant recovery has allowed the hospital to resume fulfilling the clinical needs of patients and the business needs of the organization. With this methodology developed and tested under such severe circumstances, there is confidence in our ability to maintain a flexible OR schedule during the COVID-19 pandemic. MGH is poised to reemploy these methods should the hospital encounter subsequent surges in COVID-19 cases or other emergency situations such as blizzards, hurricanes or massive power outages.

\section{Compliance with Ethical Standards}

Conflict of Interest Mr. Ryan Brumit declares that he has no conflict of interest. Mrs. Bethany Daily declares that she has no conflict of interest. Dr. Wilton C Levine declares that he has no conflict of interest.

Ethical Approval This article does not contain any studies with human participants or animals performed by any of the authors.

Informed Consent N/A

\section{References}

1. Baker, C., Elective Procedure Order [Mass.gov web site]. March 15, 2020. Available at: https://www.mass.gov/doc/march-15-2020elective-procedures-order. Accessed on: April 5, 2020, 2020.

2. Reopening Health and Human Services in Massachusetts, Retrieved from https://www.mass.gov/lists/reopening-health-and-humanservices-in-massachusetts, 2020

3. Brumit, R., MBA, Daily, B., MHA, Levine, W.C., MD, Surgical Case Deferment During a Global Pandemic. Retrieved from https:// www.ncbi.nlm.nih.gov/pmc/articles/PMC7268180/, 2020

4. Agency for Healthcare Research and Quality, HCUP CCS Fact Sheet Healthcare Cost and Utilization Project (HCUP). Retrieved from https:// www.hcup-us.ahrq.gov/toolssoftware/ccs/ccsfactsheet.jsp, 2012

5. Sud, A., Jones, M., Broggio, J., Loveday, C., Torr, B., Garrett, A., .. . Turnbull, C. (2020). Collateral damage: The impact on outcomes from cancer surgery of the COVID-19 pandemic. Annals Oncol., 31(8), 1065-1074. doi:https://doi.org/10.1016/j.annonc.2020.05.009

Publisher's Note Springer Nature remains neutral with regard to jurisdictional claims in published maps and institutional affiliations. 